\title{
Fine Structure Constant Goes Big in Spin Ices
}

\section{Inside a quantum spin ice, the constant that defines electromagnetic interactions is 10 times larger than normal, according to calculations.}

\author{
By Michael Schirber
}

$\prod$ he fine structure constant sets the strength of quantum electrodynamic (QED) interactions and is measured to be 1/137. It's hard to imagine what the Universe would look like with a different fine-structure value, but it's safe to say we humans wouldn't have made the scene. That said, an alternative "universe" might exist inside exotic materials called quantum spin ices. New calculations predict that the fine structure constant in these materials is 10 times larger than the familiar value, suggesting that quantum spin ices could offer an experimental playground for exploring QED processes that are normally suppressed [1].

Spin ices are materials with a tetrahedral lattice structure, which forces the material's magnetic spins to assume a complex pattern. At low temperatures, theory predicts that the spins form particle-like arrangements that can behave like photons, electric charges, or even magnetic monopoles (see Viewpoint: Spinning on Ice). So far, experiments have only seen hints of this quantum spin-ice behavior, so theorists are looking for new signatures that might identify it.

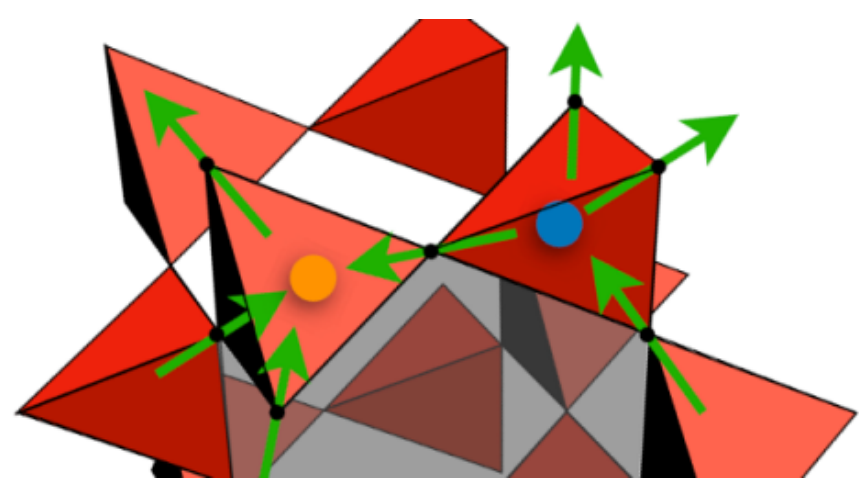

Credit: S. D. Pace et al. [1]
To study how spin-ice particles interact, Salvatore Pace from the University of Cambridge in the UK and colleagues simulated the effects of a spin-ice electric charge passing through a spin ice. They computed the strength of this elemental charge from the residual electric field energy in the ice. By combining their charge strength estimation with a previously computed spin-ice photon speed, the team found that the fine structure constant should be 1/10 in pyrochlore oxides-one of the promising quantum spin-ice candidates. The team predicts that such a strong QED interaction could lead to an observable enhancement in neutron scattering at the quantum spin-ice transition temperature.

Michael Schirber is a Corresponding Editor for Physics based in Lyon, France.

\section{REFERENCES}

1. S. D. Pace et al., "Emergent fine structure constant of quantum spin ice is large," Phys. Rev. Lett. 127, 117205 (2021). 